\title{
LO “PÚBLICO” EN LA ARQUEOLOGÍA ARGENTINA
}

\author{
THE “PUBLIC” IN ARGENTINEAN ARCHAEOLOGY \\ Virginia M. Salerno1, María Celeste Picoy², Maximiliano Tello², H. César Pinochet2,3, \\ Cecilia Lavecchia ${ }^{2,4}$ y Gabriel Moscovici Vernieri ${ }^{2,4}$
}

\begin{abstract}
Se discute el potencial de la categoría "público" para el estudio de las relaciones arqueología y sociedad. Con este fin indagamos los usos de este concepto que se registran en el campo de la arqueología argentina en los últimos quince años. Los cambios ocurridos durante este período generaron un escenario significativo para pensar las materialidades, los discursos y prácticas arqueológicas en torno a lo público. En este sentido se dieron procesos que posibilitaron no solo una mayor capacidad de acción para minorías sociales involucradas con materiales arqueológicos, sino también reformulaciones sobre el lugar de lo arqueológico en las narrativas de la historia nacional argentina. Asimismo, las "políticas de la cultura" de fines del siglo XX propiciaron la activación patrimonial de materiales y paisajes arqueológicos con fines turísticos e identitarios. En este contexto nos preguntamos: ¿qué significados se atribuyen a lo "público" en la arqueología argentina a principios del siglo XXI? ¿En qué medida estos usos se articulan con posicionamientos teóricos y debates sobre lo "público" en las ciencias sociales? ¿Qué implicancias políticas se derivan de estas conceptualizaciones?
\end{abstract}

Palabras claves: público, usos del pasado, patrimonio arqueológico, arqueología pública.

The potential of the public category is discuss in this paper for the study of the relations between archaeology and society. Pursuing this aim, the uses of this concept in Argentine Archaeology is analyzed during the last fifteen years. Within this period, social transformations generated a significant scenario for thinking materials, speeches and archaeological practices around the public category. This context has allowed a greater action capacity for social minorities involved with archaeological materials, but also reformulations over the narratives of the national history. Besides, the "politics of culture" of late 20th century led to the activation of cultural heritage materials and archaeological landscapes for tourism purposes and identity actions. Following this approach, it is worth to make some questions: what meanings are attributed to the public in Argentine Archaeology at the beginning of the 21st century? How these meanings are related to theoretical debates in social sciences? And what political implications are involved with these meanings?

Key words: Public, uses of the past, archaeological heritage, public archaeology.

Este artículo es producto de discusiones que estamos desarrollando desde el 2013 en un grupo de estudio formado a partir de la experiencia del seminario Arqueología Pública de la carrera de Antropología en la Universidad de Buenos Aires. En la actualidad nuestro grupo está integrado por estudiantes avanzados, graduados y docentes (de antropología social y arqueología). Nos planteamos interrogantes sobre la dimensión pública de la arqueología y buscamos desarrollar marcos conceptuales, ejes analíticos para una posible agenda de trabajo. En este camino, este trabajo constituye una vía para compartir preguntas y abrir al debate, antes que una propuesta de aserciones y clausuras restrictivas. Con este fin, analizamos los usos de la categoría "público" que se registran en el campo de la arqueología argentina en los últimos quince años y discutimos su potencial para el estudio de las relaciones entre arqueología y sociedad en la actualidad. En los últimos quince años se dieron una serie de cambios que propiciaron un escenario significativo para pensar las materialidades, los discursos y prácticas arqueológicas en torno a lo "público". Por estos motivos, resulta fundamental

1 CONICET, Instituto de Arqueología, Facultad de Filosofía y Letras, Universidad de Buenos Aires, Buenos Aires, Argentina. vmasalerno@gmail.com

2 Facultad de Filosofía y Letras, Universidad de Buenos Aires, Buenos Aires, Argentina. mcpicoy@gmail.com; maxitel82@hotmail.com

3 Programa de Arqueología Histórica y Estudios Pluridisciplinarios, Universidad Nacional de Luján, Luján, Argentina. hcpinochet@gmail.com

4 Instituto Nacional de Antropología y Pensamiento Latinoamericano, Ministerio de Cultura, Buenos Aires, Argentina. mariacecilialavecchia@gmail.com; gabrielmoscovici@hotmail.com 
analizar las prácticas y sentidos que se desarrollan desde la arqueología en torno a esta categoría, considerando su relación con posicionamientos teóricos y debates sobre lo "público" en las Ciencias Sociales, así como las implicancias políticas que se derivan de dichos usos.

\section{Los Debates sobre lo "Público" en Arqueología}

Desde fines del siglo XX el uso extendido de la categoría "público" dentro de la arqueología se enmarcó en una línea de trabajo denominada "arqueología pública", como concepto que identifica un amplio espectro de prácticas y temas de investigación referidos a las implicancias de la arqueología en el presente (Salerno 2013). En el contexto nacional, encontramos una primera mención a esta línea de trabajo en el XV Congreso Nacional de Arqueología Argentina celebrado en 2004. En ese encuentro se debatieron distintos $\operatorname{aspectos}^{1}$ que hacen a la dimensión social de la arqueología, dando cuenta de un proceso social y político que confluyó en el reconocimiento de estos temas como parte de los problemas a ser abordados en el campo disciplinar (Pupio y Salerno 2014). En perspectiva de la historia disciplinar, los debates sobre las relaciones entre arqueología y sociedad que contribuyeron a la legitimación del tema en nuestro país tuvieron un fuerte impulso en la década iniciada en 1980 en el marco de la apertura democrática. Estas discusiones se hicieron visibles en variados encuentros, tales como las Jornadas de Política Científica para la Planificación de la Arqueología en la Argentina realizadas en Horco Molle en 1986; el Primer Encuentro Nacional de Antropólogos en 1987, el Primer Encuentro Regional de Antropólogos de la Provincia de Buenos Aires 1988; las I y II Jornadas-Taller sobre El Uso del Pasado en 1989 y 1992, respectivamente. El compromiso social del trabajo arqueológico se abordó en términos de intervención social dirigida a la transferencia de conocimientos, a establecer relaciones con instituciones mediadoras entre comunidad e investigación -principalmente museos-y a replantear el sentido de los contenidos arqueológicos dentro de los programas escolares. Estos debates resultan fundamentales para comprender no solo los modos en que la "arqueología pública" se delineó como problema a abordar dentro del campo disciplinar, sino también los marcos éticos, conceptuales y las categorías analíticas que se establecieron para su abordaje (Daston 2000). No obstante, la legitimidad del tema se manifestó en su formulación explícita en programas institucionales y mesas de discusión de congresos nacionales recién en la década del $2000^{2}$ (Pupio y Salerno 2014; Salerno 2013). A su vez, el reconocimiento de este tema en el campo académico se articuló con discusiones que tuvieron lugar en diferentes momentos y geografías. Entre ellas, señalamos los debates anglosajones de la década de 1970 en Estados Unidos en asociación al manejo y gestión de recursos culturales (McGimsey 1972). En ese contexto, la arqueología pública designaba un campo de discusión y gestión institucional de lo arqueológico en términos de "bienes culturales" y fue debatido en el marco del Taos Conference, Nuevo México, organizado en 1988 por la Society for American Archaeology (Salerno 2013). En estos ámbitos se delineó un campo de discusión en torno a la arqueología pública que subrayó ciertos imperativos éticos de la arqueología profesional relacionados con la participación del "público" y la necesidad de desarrollar acciones de "transferencia" que impulsaran la conservación y preservación de sitios arqueológicos (Merriman 2004). A su vez, en América Latina una gran parte de las discusiones en torno a este tema se organizaron en perspectiva histórica, recuperando formulaciones críticas en torno al quehacer arqueológico (Salerno 2013). Por ello un eje central fue la discusión sobre el impacto social y político de la arqueología considerando las trayectorias coloniales y los movimientos independentistas del siglo XIX (Díaz-Andreu 1999). En esta línea, la inclusión de la categoría "público" en nuestro continente propició una ampliación semántica que cuestiona la objetivación de los materiales arqueológicos, y promueve el compromiso social con la diversidad de trayectorias históricamente excluidas e invisibilizadas (Díaz-Andreu 1999; Dussel 1994; Funari 2004; Gnecco y Ayala 2011). Se trata de una serie de debates que a pesar de tener antiguas raíces y haber sido formulados desde diferentes ubicaciones y posicionamientos teóricos, recién adquirieron visibilidad internacional en el marco de los World Archaeological Congress (WAC), iniciados en $1986^{3}$ (Salerno 2013). Estos diferentes recorridos delinean campos de acción y reflexión con marcadas diferencias teóricas y metodológicas que en la actualidad llevan a cuestionar la homogeneidad de una línea de investigación que pueda llamarse "arqueología pública" sin que 
por ello se descarte la importancia de abordar la dimensión pública del trabajo arqueológico. Por estos motivos, en este trabajo nos centramos en la discusión sobre los usos y vigencia de la categoría "público" en la actualidad.

Los debates que tuvieron lugar en la arqueología, se desarrollaron en un contexto social e histórico específico que contribuyó a la construcción de la dimensión pública de la arqueología como asunto de acción e investigación dentro del marco disciplinar. Consideramos relevante mencionar los procesos que posibilitaron una mayor capacidad de acción para minorías sociales involucradas con los materiales arqueológicos -principalmente las luchas de pueblos indígenas-; junto con reformulaciones sobre el lugar de lo arqueológico en las narrativas de la historia nacional. En Argentina, en la década iniciada en 1980 se instaló un modelo de nación pluricultural con una serie de reformas educativas y administrativas que vitalizaron las políticas culturales (Podgorny 1999). Esto produjo una nueva lógica de accionar sobre el espacio sociocultural encarnado en los Estados neoliberales. En un contexto de flexibilización del capital, de amplificación y concentración de nuevas pobrezas y riquezas, se dio un inusitado reconocimiento en las agendas políticas en derechos sobre la diversidad (derechos territoriales, legales, educativos, lingüísticos, fiscales, entre otros). El así denominado "multiculturalismo" fue retomado por el Estado nacional, mediante agendas políticas que afirman las diferencias, enfatizando lo heterogéneo y la alteridad (Zizek 2005). Estas "políticas de la cultura" propiciaron la activación patrimonial de materiales y paisajes arqueológicos con fines turísticos e identitarios (Wright 1998). Como ya mencionamos previamente, en este contexto los arqueólogos argentinos comenzaron a desarrollar una agenda de discusión en torno al lugar de la arqueología y los usos del pasado que tuvo entre sus principales temas el análisis de los aspectos vinculados a la transferencia arqueológica y las características de los contenidos arqueológicos en las escuelas (Pupio y Salerno 2014).

Particularmente, en nuestro país la crisis de 2001 implicó un cuestionamiento al modelo neoliberal y la necesidad de una inevitable transformación económica, política y social. La seguida estabilización institucional colocó al Estado, ausente en las décadas pasadas, como el partícipe activo para lograr dicha transformación. La búsqueda de fortalecimiento de la "identidad nacional" en el plano representativo retomó la "diversidad cultural" como parte de la esencia nacional estableciendo una ruptura con la imagen homogénea de identidad nacional. La representación de Argentina como un país multicultural, pluriétnico y multilingüe (Soria 2011), manifiesta una retórica que permite pensar en continuidades entre el pasado prehispánico y el presente indígena. En esta deconstrucción del discurso de lo nacional como identidad homogénea, la arqueología actualiza su discurso proclamando por identidades locales, y en pos de una reflexión metaarqueológica considera la posibilidad de convivencia con distintos pasados y tradiciones históricas. Al mismo tiempo, el cuestionamiento expresado en la reacción popular contra la profunda crisis institucional y de representación del 2001, también produjo un serio cuestionamiento sobre las narrativas históricas que explicaban ese presente en términos de orden, progreso y desarrollo democrático, sentidos que a su vez daban credibilidad y coherencia a prácticas y discursos de nuestra sociedad. Dentro de este escenario, no solo los discursos acerca del pasado colonial y prehispánico empezaron a adquirir un nuevo énfasis en el presente, además comenzaron a tener mayor visibilidad académica prácticas que llaman a la reflexividad en términos políticos, ideológicos, éticos, educativos, culturales y económicos de los intelectuales. En este contexto, el concepto "público" es utilizado por los arqueólogos argentinos con diferentes significados que refieren a grupos de personas (variados interlocutores) u objetos (bienes comunes/patrimonio arqueológico); a formas de circulación; espacios de interacción; niveles de visibilidad y acceso; y, ámbitos de gestión Estatal. Estas referencias en torno a lo "público" remiten a disputas sobre el lugar de la arqueología en el presente; su relación con las instituciones estatales, los diferentes actores involucrados y las características del patrimonio arqueológico. Al respecto nos preguntamos ¿Qué significados se atribuyen a lo "público"? ¿En qué medida estos usos se articulan con posicionamientos teóricos y debates sobre lo "público" en las Ciencias Sociales? ¿Qué implicancias políticas se derivan de estas conceptualizaciones?

\section{La Categoría "Público" en las Ciencias Sociales}

En las Ciencias Sociales, la noción moderna de "público" ha sido estudiada desde una perspectiva 
centrada en la historia de las sociabilidades, la emergencia de espacios de intercambio comunicacional y de participación política. La representación de lo social mediante lo público y lo privado permite definir espacios de competencia para distintas actividades (económicas, políticas, culturales, sociales, etc.), así como espacios de interacción donde se reproducen y construyen distintas normatividades. Es decir, lo público es una categoría de clasificación social que incluye sentidos múltiples y diferenciados vinculados a escenarios de acción de los actores sociales y políticos; además constituye un punto de referencia socioterritorial (Iazzeta 2008; Moore 1984; Ortiz 2002). En el transcurso de los siglos y en geografías específicas, este orden se fue redefiniendo. El interrogante sobre el modo en que se define y organiza lo público ha sido la guía de variados estudios a lo largo del siglo XX. Una gran parte de ellos se configuraron en relación con la historia de los Estados-Nación, otorgando a esta categoría significados políticos en asociación con el concepto de ciudadanía (Guerra y Lemperiere 1998; Iazzeta 2008), otros abordajes consideraron lo público desde sus implicancias en procesos de clasificación social (Chartier 2007; Moore 1984).

Sin buscar hacer un recorrido exhaustivo, lo que nos interesa mostrar es que existen diferentes marcos interpretativos desde donde significar lo público en oposición a lo privado. Estos enfoques problematizan preguntas como las siguientes: ¿qué aspectos de la vida social pueden considerarse como parte de un orden y otro?, y ¿de qué manera estas diferencias operan en la organización social y distribución de poder? Desde el modelo económico liberal lo público aparece asociado a la administración estatal y lo privado a la economía de mercado (Gil Fernández 2005; Guerra y Lemperiere 1998; Habermas 1981). Desde un enfoque centrado en la virtud republicana, lo público referencia a una comunidad política y ciudadana, respecto de lo privado encarnado en el Estado (Iazzeta 2008). Por su parte, la discusión en torno a lo público-estatal y no-estatal recupera la metáfora espacial para designar ámbitos de producción de cultura e identidad, así como espacios de representación social, de imposición de sentidos y construcción de hegemonía (Fraser 1999). Así por ejemplo desde una perspectiva feminista, se ha observado cómo el espacio público, encarnado en el circuito laboral, aparece representado como un campo de conquista respecto de un dominio privado asociado a la esfera doméstica (Pateman
1996). Lo privado como aquello que se identifica con lo individual -secreto- y lo público a aquello vinculado con lo colectivo - visible- (Bobbio 1989; Gil Fernández 2005; Guerra y Lemperiere 1998; Iazzeta 2008).

Desde una perspectiva histórica y sociológica, la discusión sobre la dicotomía público-privado se organiza a partir de la pregunta respecto de cómo se origina esta distinción y cuáles son sus efectos. En este camino, Norbert Elias (1996) explicó de qué forma se produjo un proceso de privatización de conductas sociales, dando lugar a la identificación de Occidente con la "civilización". Este estudio da cuenta de los complejos vínculos entre procesos de control y autocontrol de comportamientos que a la vez establecen las fronteras entre lo público y lo privado en escala mayor. De igual forma, Giddens (1995) argumenta que estas distinciones permiten legitimar el comportamiento social y político, publicitando objetivamente no solo todo aquello que es entendido como parte de lo social sino también todo aquello que es parte de la vida íntima y emocional.

Estos enfoques consideran la dicotomía como parte de los criterios sociales, definidos históricamente, utilizados para diferenciar y clasificar el comportamiento de personas y grupos en términos de visible/oculto, normal/anormal, bueno/malo, alto/bajo, central/periférico, legítimo/ ilegítimo. La importancia de estas falsas dicotomías reside en que las mismas organizan categorías sociales a partir de la cuales nos desenvolvemos en la actualidad. En su configuración intervienen sujetos sociales con diferentes intereses y capacidad de acción, vinculados con distintos espacios de sociabilidad -como por ejemplo el Estado, las empresas, los sindicatos, los movimientos sociales, las organizaciones sin fines de lucro, las empresas y los medios de comunicación-. Asimismo, estos procesos deben pensarse entrelazados con los procesos de globalización de las tecnologías de comunicación, la organización económica y financiera de las empresas, la reestructuración transnacionalizada de las comunidades de ciudadanos y consumidores (Ortiz 2002).

\section{El Uso de la Categoría "Público" en la Arqueología Argentina}

Para abordar los interrogantes planteados al inicio de este trabajo, analizamos el modo en que se utiliza el concepto "público" en diferentes producciones 
elaboradas por arqueólogos y especialistas vinculados con la gestión del conocimiento arqueológico en Argentina. Con este fin, se seleccionaron una serie de textos (publicaciones en revistas científicas, presentaciones en congresos, páginas web elaboradas por grupos de investigación, presentaciones de programas institucionales, trabajos de divulgación) que incluyeran el uso de la categoría "público". De este modo, nuestro trabajo desarrolla una mirada transversal acerca de los usos de la categoría "público" al incluir el análisis tanto de artículos publicados en revistas y reuniones académicas como de publicaciones orientadas a destinatarios no necesariamente especializados, dado que se trata de discursos con diferentes propósitos comunicativos. Esto nos permitió abordar el análisis sobre el uso de la categoría reconociendo distintos discursos elaborados por los mismos actores, cuyas diferencias remiten, en muchos casos, a los límites y alcances de cada contexto enunciativo. La consideración de estos diferentes géneros discursivos fue un aspecto central del análisis que nos permitió recuperar un amplio espectro de prácticas asociadas con el uso de la categoría que se relacionan con la investigación, la ética, la comunicación, la conservación, la educación y la gestión. El análisis interrelacionado de estos diferentes textos se llevó a cabo identificando "grupos productores/equipos" para poder considerar las relaciones entre las formulaciones teóricas en torno a la construcción de problemas, sujetos, espacios e implicancias de la categoría "público" y su puesta en práctica en proyectos discursivos específicos. En tanto las formulaciones teóricas y su puesta en práctica constituyen dos dimensiones ineludibles de la acción social que comportan la elaboración de sentidos en torno a la categoría "público" por parte del mismo grupo productor (Lahire 2006). Como un aspecto que se desprende de este análisis integrado, es importante destacar que dentro de cada equipo se observaron diferentes posicionamientos entre los autores respecto del tema. Además, de acuerdo con los objetivos de cada grupo, este tipo de temas es abordado de forma diferencial (en términos de tiempo y recursos dedicados al mismo) en relación con el resto de temas que involucran el proceso de elaboración de conocimiento arqueológico en cada equipo. Queda pendiente en este análisis indagar el modo y la intensidad en que estos aspectos inciden en los productos finales y en muchas ocasiones en la elaboración de discursos contradictorios que representan al mismo grupo.
En total analizamos 61 textos que corresponden a 19 grupos de los cuales solo seis se identifican explícitamente con la arqueología pública, en términos de "línea de trabajo y/o subcampo disciplinar", o en términos de "prácticas de arqueología pública". Mientras que el resto utiliza el concepto "público" sin enmarcar la propuesta dentro de líneas específicas. Como ya mencionamos, esto no implica para nosotros atribuir a la arqueología pública una única, homogénea y universal línea de investigación en Argentina. Si bien identificamos campos de acción y reflexión muy variables en función de contextos locales y de tradiciones académicas de las arqueologías que se desarrollan en cada lugar, consideramos que esto no descarta la posibilidad de abordar analíticamente la dimensión pública del trabajo arqueológico. Las adscripciones semánticas explícitas o implícitas de la categoría "público" fueron consideradas a partir de su contexto de enunciación, comprobando en muchos casos la existencia de usos polisémicos de la categoría.

En la Tabla 1 se detalla la distribución regional de los grupos analizados, con esta selección buscamos generar una perspectiva representativa que incluyera la diversidad de espacios donde se desarrolla la investigación arqueológica en el país. Cabe destacar que en el amplio territorio argentino existen vastas diferencias en las características de la materialidad arqueológica así como en la presencia de actores claves, principalmente pueblos originarios, que en la actualidad demandan una mayor participación en la gestión de los materiales arqueológicos. En el análisis de los casos seleccionados consideramos las diversas formas en que el trabajo arqueológico se articula en espacios concretos y a partir de variadas trayectorias académicas.

En el análisis y procesamiento de los textos, trabajamos con un programa informático específico para el tratamiento de datos cualitativos (QDA

Tabla 1. Distribución regional de las investigaciones de los grupos analizados.

Regional distribution of analyzed research groups.

\begin{tabular}{cc}
\hline Región & $\%$ \\
\hline Noroeste & 23,0 \\
Sierras Centrales & 7,7 \\
Cuyo & 15,4 \\
Patagonia & 15,4 \\
Pampa & 30,8 \\
Noreste & 7,7 \\
\hline
\end{tabular}


Miner Lite). A partir de este programa realizamos un primer ordenamiento de los datos que nos permitió efectuar después una codificación de los mismos de acuerdo con nodos pertinentes. Para ello elaboramos un árbol de categorías y subcategorías que fueron establecidas a partir de un procedimiento mixto que incluyó un abordaje deductivo, organizado mediante categorías y subcategorías que habían sido previamente establecidas por nuestros interrogantes, con un enfoque inductivo, que nos permitió incluir categorías elaboradas a medida que se analizaron los datos. De esta manera, los principales ejes de análisis establecidos previamente, referidos al uso de la categoría público en referencia a objetos, a sujetos y a espacios, se enriquecieron con la identificación de prácticas específicas, de una amplia gama de categorías asociadas al concepto junto con la descripción de supuestos disciplinares, tensiones y propósitos que movilizan las acciones.

En este punto quisiéramos destacar que a pesar de nuestras expectativas no identificamos diferencias claras en torno a la categoría "público" de acuerdo con las disímiles situaciones territoriales en que se desenvuelve el trabajo arqueológico. Al contrario, hemos identificado un alto grado de homogeneidad en relación con los temas y prácticas abordadas respecto de la categoría "público". Los temas referidos en los textos analizados remiten a la comunicación de conocimiento científico en un sentido amplio. Incluyendo la reflexión y presentación de experiencias concretas asociadas con la divulgación, la extensión universitaria, el trabajo en el marco de voluntariados universitarios, la elaboración de materiales de comunicación y trabajos en articulación con instituciones locales (museos, escuelas, municipios). En estos trabajos existen disímiles posicionamientos teóricos que incluyen tanto reproducciones del llamado modelo del déficit (Cortassa 2012) como posicionamientos críticos que aspiran a desarrollar propuestas de cogestión con actores locales. A pesar de estas diferentes matrices teóricas encontramos un alto grado de homogenidad en el tratamiento del concepto público.

Otro tema ampliamente abordado refiere a la gestión del material arqueológico en el marco de activaciones patrimoniales, principalmente asociadas a desarrollos turísticos y/o problemas de conservación. Aquí se incluyen estudios sobre "imaginarios" en torno al patrimonio arqueológico; proyectos de registro y mapeo de repertorios patrimoniales, junto con planteos críticos a la noción de patrimonio arqueológico. Los planteos críticos se desarrollan tanto desde una perspectiva histórica como desde la denuncia de situaciones de conflicto que tienen lugar en la actualidad y que dan cuenta de demandas de minorías sociales (principalmente pueblos originarios pero no de forma excluyente, también aparecen conflictos con coleccionistas, pobladores locales, empresas, etc.) para participar en la interpretación y gestión de los materiales arqueológicos. De estas propuestas se derivan en algunos casos proyectos que buscan generar instancias de participación de distintos actores sociales en la gestión del patrimonio arqueológico. Finalmente, encontramos solo en el caso de tres equipos (lo que corresponde al 15\% del total) propuestas de investigaciones que abordan procesos de apropiación y resignificación del material arqueológico, asî como las relaciones entre sujetos y materiales arqueológicos en el presente.

\section{Investigar, Conservar y Divulgar}

A pesar de las amplias formulaciones conceptuales que acentúan el carácter dinámico, histórico y negociado de lo público en las ciencias sociales, en los registros relevados dentro del campo de la arqueología argentina predominan usos reificados del mismo. Esto es, utilizan la noción de público para describir los materiales arqueológicos en términos patrimoniales. Como parte de los "bienes públicos", el patrimonio arqueológico es entendido como un objeto perteneciente a la sociedad en su conjunto y configurado por/en un corpus normativo que recupera los sucesivos sentidos otorgados en el marco de la organización de competencias del Estado nacional argentino. Se trata de una noción objetivada en la legislación (leyes 9080 de 1913 y ley 25743 de 2003), donde el "interés general" es representado mediante los valores y los intereses científicos emulados como universales. El mismo remite a la información que puede obtenerse sobre las poblaciones que habitaron el actual territorio argentino, inclusive en épocas anteriores a la conformación del Estado Nacional.

Esta representación del todo social, presente en el corpus legislativo sobre patrimonio, implica desparticularizar y despolitizar el pasado para integrarlo como parte de una historia de propiedad colectiva ausente de conflictos (Crespo 2005). Desde esta perspectiva universalista se conceptualizan las relaciones entre arqueólogos -materiales 
arqueológicos- y sociedad civil, estableciendo un dominio público a partir del cual se justifica la necesidad de determinado tipo de prácticas. A continuación se transcriben algunos ejemplos:

Un plan de consenso entre los diferentes actores sociales involucrados, que permita fundar acciones de utilización responsable del patrimonio arqueológico. De esta manera se generan estrategias de integración entre el conocimiento arqueológico, el turismo y el sistema educativo, que permiten la revalorización de los recursos patrimoniales, a los fines de promover la conservación y adecuado manejo del Patrimonio Arqueológico (Grupo 2, artículo académico).

Es a través de estos materiales que vivenciamos nuestra propia historia y podemos construir Identidad (Grupo 8, artículo de divulgación).

La realización de cursos y talleres orientados a los diferentes públicos interesados, permitiendo de esta manera que los habitantes de las localidades involucradas se acerquen a su pasado prehispánico [...] Si bien son los Arqueólogos quienes trabajan con estos restos y su contexto, es responsabilidad de todos conservarlos, debido a que son el único medio a través del cual podemos conocer nuestra historia local, regional y nacional (Grupo 1, artículo de divulgación).

En estas formulaciones se organiza un campo de acción dentro del Estado para la conservación y gestión de los bienes comunes. Una de las implicancias de este proceso de institucionalización de los materiales es que recontextualiza las representaciones del pasado y su materialidad por fuera de la vida cotidiana de las personas y de los contextos locales específicos para posicionar sujetos expertos que prescriben límites, establecen prácticas y unifican criterios en torno a los materiales. Son estos sujetos expertos, "especialistas", "funcionarios", "arqueólogos", los encargados de gestionar los materiales arqueológicos y de transmitir su importancia a la sociedad, el "público-ciudadano", "audiencia", "pobladores locales", en términos de concientizar, poner en valor, hacer divulgación. En este marco de relaciones se define una agenda de problemas que se organiza mediante formas legítimas de intervención y relación con los materiales arqueológicos. De esta manera se definen los usos adecuados del patrimonio.

A su vez, se reconoce a la "ciudadanía" como el sujeto-destinatario cuya presencia es necesaria para dar sentido a las acciones que se llevan a cabo, ya que sin destinatarios ni la conservación ni la difusión tendrían lógica. Esto en ocasiones es planteado como una forma de legitimidad de la actividad; en otras como un "deber ser" del buen profesional cuyos procesos no estarían completos sin incluir la tríada "investigar, conservar, divulgar" que es el legado de las definiciones de la UNESCO:

La estrategia de socialización del proyecto y sus resultados entre la comunidad local es otro de los ejes del proyecto, ya que es la única forma de promocionar un sentido de pertenencia de la comunidad respecto a su pasado y legitimar, en última instancia, nuestros objetivos (Grupo 15, artículo académico).

En este sentido pensamos que es importante la difusión del conocimiento científico disponible para cada región, que, aunque escaso en la mayoría de los parajes donde no se realizaron estudios sistemáticos, significa mucho para los habitantes locales, quienes tienen un vacío en relación con su pasado. Esta socialización del conocimiento, amén de aportar a las comunidades locales, ayuda a difundir y validar la labor realizada por arqueólogos y antropólogos, dando respuesta a una de las demandas más recurrentes vinculada con la búsqueda del pasado (Grupo 17, artículo académico).

De igual forma, en algunos casos se plantea la gestión patrimonial como una forma de contribuir al desarrollo local, lo que asume una relación directa entre material arqueológico como bien patrimonial y recurso común con valor económico/simbólico en los siguientes términos:

Realizar una propuesta que regule y articule a medio plazo las acciones necesarias para convertir el yacimiento en un recurso para el desarrollo [...] En este documento se materializarán y concretarán diferentes aspectos estratégicos de cara a 
la recuperación integral del yacimiento, como son la construcción de un discurso histórico, la conservación física de los testimonios arqueológicos, la estrategia social de compromiso con el entorno o la creación de una sede física de interpretación y encuentro (Grupo 15, artículo académico). El patrimonio cultural debe ayudar al progreso humano, y en este proceso es fundamental su identificación y valoración, sobre todo en regiones poco favorecidas, abriéndose un debate sobre cómo aprovecharlo de una forma útil y responsable, que asegure su rentabilidad social, cultural y también económica (Grupo 17, artículo académico).

\section{Salir a lo Público}

Otros usos reificados del concepto público hacen referencia a un espacio físico o simbólico en donde ocurre, se accede, se construye o se puede circular. En esta perspectiva, el concepto funciona menos como entidad directamente observable que como categoría descriptiva de un campo heterogéneo de comunicación entre arenas discursivas y entidades diversas. Así se hace referencia a agentes en sectores públicos, sitios arqueológicos públicos, áreas disciplinares o institucionales que abordan la cuestión pública, discursos que tienen que ser ubicados en lo público. El caso más extendido de este campo de significados lo encontramos en las nociones que plantean como una necesidad de la arqueología el "salir" y generar "apertura", con el fin de formar parte y afectar un espacio público en el que tienen lugar debates y discusiones relativas a su quehacer. Nos interesa destacar que el "salir a lo público" implicaría un desplazamiento que se plantea como parte de un proceso coyuntural, relacionado tanto con demandas de actores que no formarían parte del ámbito académico, científico y profesional como con el debate interno sobre la función social de la arqueología en el presente:

Los arqueólogos necesitamos "salir del sitio", dar un espacio a otras miradas que trascienden el valor científico y los aspectos vinculados con la conservación del patrimonio (Grupo 5, artículo académico). El objetivo de toda nuestra producción, manteniendo los estándares y la rigurosidad que demanda el mundo académico -es necesariamente involucrarse con las respuestas y los intereses de la comunidad en relación a su pasado. Trabajamos en un equilibrio permanente entre la construcción de fundamentos teóricos para aplicar ciertas metodologías $[\ldots]$ pero al mismo tiempo tenemos que generar una información correctamente codificada o traducida a la comunidad, que es la principal usuaria de los datos históricos y arqueológicos (Grupo 1, artículo académico).

Estos usos suponen como punto de partida pensar a la arqueología por fuera de lo público, es decir, como ámbito o esfera de grupos privados. De este modo se establece un reconocimiento implícito de un ámbito privado y particular asociado con la producción de conocimiento que incluiría el diseño de investigación, el trabajo de campo, el análisis en laboratorio, la publicación de artículos científicos y la presentación en reuniones académicas, entre otros. Dentro de esta perspectiva, encontramos una variedad de usos en los que se reproduce una perspectiva cientificista y conservadora del valor del patrimonio arqueológico. A partir de ello se naturaliza la intervención en lo público por el valor intrínseco de lo que la arqueología podría aportar al "bien común". En estos usos la "apertura a lo público" estaría dada por acciones comunicativas generalmente presentadas en términos de "transferencia", "extensión", "divulgación" o "difusión", cuyo objetivo es poner en circulación ante una determinada audiencia parte de los resultados de la investigación arqueológica y de esta forma generar "visibilidad" y "accesibilidad". En este camino se plantean cuestiones referidas al modo en que se organiza esta visibilidad, ya sea mediante la apertura de sitios "al público" a través de planes de manejo o mediante la puesta en circulación de los conocimientos aportados por los arqueólogos. Este tipo de propuestas reproducen el modelo de déficit (Cortassa 2012). En el mismo se asume que los destinatarios del mensaje tienen un déficit de conocimiento científico que puede ser subsanado por la comunicación científica. A su vez, se da por supuesto que los conocimientos puestos a circular, transferidos al colectivo de "destinatarios pasivos", tienen como efecto la generación de valoraciones positivas sobre la arqueología y los materiales que ella estudia. 
A su vez, en las propuestas de intervenciones en el espacio público los ámbitos escolares son uno de los escenarios privilegiados en todas las regiones del país. En menor medida se mencionan medios masivos, museos, sociedad civil, instituciones locales en general. Los ámbitos escolares se asumen como el principal mediador entre los conocimientos arqueológicos y la comunidad. En torno a ellos abundan trabajos sobre características de recursos didácticos, análisis de la forma en que se abordan los contenidos escolares, encuestas a docentes para conocer representaciones y a partir de los cuales se plantea la intervención en estos espacios. A pesar de estos amplios y diversos enfoques, solo en escasas ocasiones se reconoce el complejo proceso creativo que tiene lugar en las instituciones escolares. En cambio, las mismas son mayormente caracterizadas como ámbitos de reproducción y transmisión uniforme.

En conjunto, nos llama la atención que en estas argumentaciones lo que se abre y pone a circular es conocimiento y objetos -que son mal comprendidos, resignificados, apropiados, negociados-, lo que implica una mirada escindida de las relaciones institucionales y personales en las que se enmarca la construcción de un ámbito público.

\section{La Dificultad de la Crítica}

Para terminar esta presentación, nos interesa subrayar que no solo son escasas las formulaciones críticas a las nociones objetivadas del patrimonio arqueológico, sino que además las mismas se presentan con discursos contradictorios, ya sea en las elaboraciones de diferentes sujetos del mismo equipo o dentro de un mismo texto argumentativo. Las contradicciones se expresan en la afirmación de nociones objetivadas del patrimonio arqueológico y la declamación de acciones tendientes a su conservación, junto con planteos que relativizan su valor universal y que, en consecuencia, dejan sin efecto los planteos previos. En pocos casos se menciona la presencia de conflicto, y cuando se lo hace este aparece en términos de tensión en las formas en que participa la sociedad civil y en la relación con actores específicos que reclaman un dominio exclusivo sobre el "bien público". Estas menciones devienen en la discusión de la noción de patrimonio y la necesidad de incluir a otros actores en su proceso de valoración. En este sentido, el uso de la categoría público podría estar indicando la necesidad de abordar aspectos que hacen al trabajo arqueológico y que no necesariamente son considerados como tales. Por ejemplo, en esta línea de argumentación se incluyen algunos trabajos que describen lo público como un espacio en el que irrumpen controversias que refieren a relaciones de poder, específicamente conflictos de legislación con grupos que expresan diferentes valoraciones, objetivos y racionalidades. En estos casos, lo público se propone como espacio de diálogo, de sociabilidad y de negociación en el que los arqueólogos se posicionan como mediadores frente a diferentes colectivos de la sociedad civil, el Estado y el mercado. Sin embargo queda sin discusión la concepción universalista del valor del "bien común", supuesto en el que se sostiene la validación del rol mediador del arqueólogo. De esto resultan propuestas de diálogo y coparticipación que no consideran las trayectorias diferentes de los actores involucrados ni el marco de relaciones desiguales en que los diálogos se organizan. Entendemos que esta escasa presencia de miradas críticas puede ser interpretada como un proceso de olvido y/o desconocimiento de los procesos históricos que dieron lugar a la conformación del patrimonio arqueológico en términos de "bienes públicos" en el marco del Estado Nacional. Olvido y/o desconocimiento que es requerido para la elaboración de miradas naturalizadas del concepto de patrimonio arqueológico como "bien público" y, por estos motivos, resulta funcional a una estructura de relaciones que legitima el rol preponderante de la actividad científica.

A su vez, tanto desde las perspectivas críticas como naturalizadas, en el plano discursivo se promueven el mismo tipo de prácticas (institucionalizar, divulgar, intervención en ámbitos de enseñanza formal y no formal, puesta en valor de sitios, realización de talleres participativos). Esto nos lleva a preguntarnos en qué medida diferentes acciones son nombradas de la misma manera. De igual forma, en ambos casos, perspectivas críticas y naturalizadas, se observa una ausencia del campo de sentidos que asocian la dicotomía abierto-cerrado con las discusiones que se vienen dando en el campo de las ciencias sociales. Entendemos que esta ausencia contribuye a correr del eje de discusión a la dimensión política en la organización de lo público y reducirlo a un problema de visibilidad/accesibilidad.

Es interesante recalcar que buena parte de la dicotomía entre lo público y lo privado proviene no solo de los ámbitos de formación profesional de los arqueólogos, sino también de la relación 
cotidiana que establecemos con los "otros" durante el trabajo de campo - pueblos originarios, escuelas, personas del pueblo interesados en su pasado, coleccionistas, etc-. En este sentido, aunque de forma contradictoria, las tensiones y conflictos con actores que son categorizados como parte de lo "público" es uno de los aspectos que el uso de la categoría contribuye a visualizar. Esto resulta fundamental no solo en el análisis de la vigencia del discurso del multiculturalismo en Latinoamérica y las demandas de los pueblos originarios respecto de la arqueología (Flores y Acuto 2015), sino también en las disputas que se dan con "estudiosos", "aficionados" y "coleccionistas" que actualmente son excluidos por no ser "profesionales". Si bien se trata de actores y procesos con trayectorias sumamente distintas cuyo abordaje merece un artículo aparte, buscamos señalar que las mismas están atravesadas por los límites -contingentes y políticos- entre lo público y lo privado en la práctica arqueológica.

En este sentido, aunque establecemos que la arqueología, en tanto disciplina científica, ocupa un lugar hegemónico en la producción y enunciación de conocimientos del pasado (y por lo tanto del presente), debemos tener en cuenta que muchos conocimientos en relación con lo "arqueológico" y sus formas de narración son producidos también por ámbitos no académicos. De esta forma, comunidades locales, pueblos indígenas y distintos sujetos sociales (como coleccionistas, aficionados a la arqueología y demás) generan sus propias representaciones sociales del pasado a partir de intereses presentes mediante el interjuego entre sus propias experiencias de vida, los discursos sociales, las interpretaciones acerca de sí mismos y los otros sociales con los cuales se relacionan. Estas narraciones arqueológicas se encuentran atravesadas por múltiples voces, muchas veces en relación de tensión y antagonismo, que nos llevan a reflexionar y tener presente que la arqueología en tanto "ciencia social" no es la única que intenta hegemonizar significados y relaciones entre el pasado y el presente a partir de los materiales.

\section{Consideraciones Finales}

En el recorrido anterior observamos que la noción de público es utilizada por equipos de profesionales de la arqueología argentina principalmente como categoría descriptiva, ya sea para designar grupos de personas (audiencias, ciudadanos) u objetos (bienes comunes, patrimonio arqueológico) así como en referencia a metáforas espaciales en términos de acceso común o ámbito de interacción. Se trata de usos descriptivos y asociados a casos concretos esquematizados en esta síntesis con fines analíticos y en pos de priorizar el debate, generar preguntas sobre las implicancias de este tipo de usos.

En este proceso subrayamos una serie de ejes asociados con los significados que se otorgan a lo público y al lugar de los arqueólogos en su configuración. A pesar de nuestras expectativas, encontramos una amplia uniformidad en la que predomina un uso de la categoría en términos descriptivos que desconocen el amplio espectro de discusiones desarrollado en las ciencias sociales en torno a la misma y que han formado parte de debates dentro del campo de la arqueología pública (Salerno 2013). Al respecto se podría argumentar que no se trata de una categoría central en las propuestas desarrolladas por parte de los arqueólogos, no obstante, es utilizada para justificar y definir estrategias de intervención social, acciones concretas que se postulan dentro de una dimensión ética en la que prevalecen ideas claras sobre el compromiso en la transmisión de conocimiento y la construcción del saber. Inclusive, estos usos descriptivos aparecen en propuestas enmarcadas dentro de la "arqueología pública" como línea de investigación y acción.

Estos usos de la categoría posibilitan establecer como punto de partida un conjunto de valores y normas objetivadas en torno a lo "público" que legitiman el rol del profesional e invisibilizan las relaciones desiguales e históricas en que las mismas se forjaron. De esta manera no se reconoce el lugar de los profesionales de la arqueología en la configuración de "lo público", en cambio se asume para los arqueólogos un rol de imparcialidad y mediación en tanto su intervención representa el asumido "interés común" ante diferentes sujetos sociales. Llevado a un extremo resulta interesante que si la arqueología tiene un rol social o público respecto de hacer cumplir los derechos culturales a partir de reconocer en el otro un ciudadano del Estado, entonces, frente a grupos que reclaman autonomía política ante el Estado nacional el rol público de la arqueología se vuelve intrascendente. Con este ejemplo lo que buscamos argumentar es que cuando se asume como dado que la arqueología en tanto actividad científica conlleva en sí misma un rol 
social o público de valor "universal", se legitiman varias acciones y sujetos a la vez que se invalidan y desconocen otras posibilidades. La principal consecuencia de este tipo de usos es que se oculta la dimensión política del quehacer arqueológico.

Para abordar este problema resaltamos la necesidad de una ética reflexiva que permita dar cuenta del lugar de enunciación en la generación de conocimiento: pensar el lugar del arqueólogo e introducir la dinámica del conflicto como parte de los trabajos. La actividad arqueológica está atravesada por múltiples contradicciones debido a que es parte de ámbitos estatales y es heredera de una historia, en nuestro caso, erigida a partir de relaciones coloniales. De esto resultan una serie de conflictos que se dan no solo en las relaciones entre arqueólogos y comunidades, sino también hacia el interior del ámbito académico -en la formación de profesionales- y en el mercado laboral como, por ejemplo, la arqueología de contrato. Estas situaciones son escasamente asumidas, excepto algunos casos puntuales que se dan en el marco de situaciones conflictivas entre arqueólogos y actores locales. Cabe preguntarse en qué medida las contradicciones de la práctica median la construcción del conocimiento y las relaciones que se establecen en los ámbitos locales.

Para finalizar este trabajo quisiéramos subrayar la utilidad de lo "público" como herramienta analítica que permite significar y conceptualizar relaciones sociales, históricas y cambiantes. Lo "público" es principalmente un espacio de disputa de significados mediante el cual se establecen fronteras sociales a partir de la representación dicotómica (público/ privado). Entendida de esta manera, esta noción permite introducir la dinámica del conflicto y desplazar la perspectiva desde entidades objetivadas (sujetos, objetos, espacios) hacia los procesos de desigualdad y exclusión que atraviesan la formación de dichos espacios.

Agradecimientos: Se agradece a Lucio Menezes Ferreira, Magdalena Frère y Milva Umaño por sus comentarios. A los evaluadores cuyas observaciones contribuyeron a enriquecer las discusiones de este artículo. El desarrollo de esta propuesta se enmarcó en los proyectos UBACyT 2011- 2014 01/W134 y PICT 2014-0643.

\section{Referencias Citadas}

Bobbio, N. 1989. Estado, Gobierno y Sociedad. Por una Teoría General de la Política. Fondo de Cultura Económica, México, D.F.

Chartier, R. 2007. Lo público y lo privado. Construcción histórica de una dicotomía. Revista Co-herencia 4:65-81.

Cortassa, C. 2012. La Ciencia Ante el Público. Eudeba, Buenos Aires.

Crespo, C. 2005. "Que pertenece a quien": procesos de patrimonialización y pueblos originarios en Patagonia. Cuadernos de Antropología Social 21:133-149.

Daston, L. 2000. Biogaphies of Scientific Objects. The Univesity of Chicago Press, Chicago.

Díaz-Andreu, M. 1999. Nacionalismo y arqueología: del Viejo al Nuevo Mundo. Revista do Museu de Arqueologia e Etnologia, Anais da I reuniao Internacionacional de Teoria Arqueologica na America do Sul 3:161-180.

Dussel, E. 1994. 1492. El Encubrimiento del Otro. Hacia el Origen del "Mito de la Modernidad". UMSA, La Paz.

Elias, N. 1996 [1966]. La Sociedad Cortesana. Fondo de Cultura Económica, México, D.F.

Flores, C. y F. Acuto 2015. Pueblos originarios y arqueología argentina. Construyendo un diálogo intercultural y reconstruyendo la arqueología. Intersecciones en Antropología 16:179-194.
Fraser, N. 1999. Repensando la esfera pública. Una contribución a la crítica de la democracia actualmente existente. Revista Ecuador Debate 46:139-173.

Funari, P. 2004. Public archaeology in Brazil. En Public Archaeology, editado por N. Merriman, pp. 202-210. Routledge, Londres y Nueva York.

Gero, J. 2000. Why? and Whither? WAC. World Archaeological Congress Bulletin 12:55-63.

Giddens, A. 1995. La Constitución de la Sociedad. Bases Para una Teoría de la Estructuración. Amorrutu Editores, Buenos Aires.

Gil Martínez, F. 2005. Tecnología y esfera de lo público en Jürgen Habermas. Revista CTS 5:141-152.

Gnecco, C. y P. Ayala (eds.) 2011. Indigenous People and Archaeology in Latin American. Left Coast Press, Walnut Creek.

Guerra, F.X. y A. Lemperiere 1998. Los Espacios Públicos en Iberoamérica. Ambigüedades y Problemas. Siglos XVIII-XIX. Centro Francés de Estudios Mexicanos y Centroamericanos y Fondo de Cultura Económica, México, D.F.

Habermas, J. 1981 [1962]. Historia y Crítica de la Opinión Pública. La transformación estructural de la vida pública. Editorial Gustavo Gili, México, D.F.

Iazetta, O. 2008. Lo público, lo estatal y la democracia. Revista Iconos 32:49-60. 
Lahire, B. 2006. El Espíritu Sociológico. Manantial, Buenos Aires.

McGimsey, Ch. 1972. Public Archaeology. Seminar Press, Nueva York.

Merriman, N. 2004. Introduction: Diversity and dissonance in public archaeology. En Public Archaeology, editado por N. Merriman, pp. 1-18. Routledge, Londres y Nueva York.

Moore, B. 1984. Privacy: Studies in Social Cultural History. ME Sharp Ink, Nueva York.

Ortiz, R. 2002. Globalización y esfera pública. Entre lo nacional y lo transnacional. Revista Signo y Pensamiento XXI:68-81.

Pateman, C. 1996. Críticas feministas a la dicotomía público/ privado. En Perspectivas Feministas en Teoría Política, compilado por C. Castells, pp. 31-50. Paidós, Barcelona.

Podgorny, I. 1999. Arqueología de la Educación. Textos, Indicios, Monumentos. La Imagen de los Indios en el Mundo Escolar. Sociedad Argentina de Antropología, Buenos Aires.
Pupio, A. y V.M. Salerno 2014. El concepto de patrimonio en el campo de la Arqueología argentina. Análisis de los trabajos presentados en los congresos nacionales de Arqueología (19702010). Revista Intersecciones en Antropología 15:115-129.

Salerno, V.M. 2013. Arqueología pública. Reflexiones sobre la construcción de un objeto de estudio. Revista Chilena de Antropología 27:7-37.

Soria, S. 2011. La Reinvención de la Nación en la Argentina actual: estado relato nacional y pueblos indígenas. Nómadas 34:214-228.

Wright, S. 1998. The politicization of "Culture". Anthropology Today 14:7-15.

Zizek, S. 2005. Multiculturalismo o la lógica cultural del capitalismo multinacional. En Estudios Culturales. Reflexiones sobre el Multiculturalismo, editado por F. Jameson y S. Zizek, pp. 137-188. Paidós, Buenos Aires.

\section{Notas}

1 Entre ellos pueden mencionarse: la percepción del público sobre los sitios arqueológicos, las representaciones sociales respecto del patrimonio, la relación entre los arqueólogos y los pueblos originarios y su participación en el proceso de investigación, entre otros.

2 La primera mención a esta línea de trabajo en Argentina, aparece en una mesa del XV Congreso Nacional de Arqueología Argentina celebrado en el 2004. También pueden mencionarse como ejemplos la Mesa de discusión "Arqueología en la práctica" de las Jornadas de jóvenes investigadores del INAPL en 2012; el simposio "Arqueología Pública" que tuvo lugar en 2013 en el XVIII Congreso Nacional de Arqueología
Argentina con una gran cantidad de participantes; el Grupo de Trabajo "Patrimonio, arqueologías contemporáneas y politización: Debates en torno a estrategias de intervención en la esfera pública" desarrollado en el XI Congreso Argentino de Antropología Social en 2014, entre otros.

3 En estos congresos se abordaron temas relacionados con las dimensiones éticas de la arqueología y el rol del profesional en la sociedad; las relaciones de poder al interior de la disciplina, la presencia de diferentes miradas y usos de las evidencias del pasado, la educación y la diversidad de formas de construir narrativas sobre el mismo (Funari 2004; Gero 2000; entre otros). 